\section{Multifocal electroretinography changes in patients on ethambutol therapy}

\begin{abstract}
Purpose To evaluate the multifocal electroretinography (mfERG) changes in patients on ethambutol therapy.

Methods A cross-sectional observational study of 17 visually asymptomatic patients receiving antituberculosis therapy with ethambutol. Patients underwent complete ophthalmic examination and mfERG
\end{abstract} recordings. The first-order mfERG N1 and P1 response amplitudes and implicit times of six concentric rings were analysed and compared with 17 normal age-similar controls.

Correlation analyses were performed between the patients' mfERG parameters with parameters of ethambutol usage (daily dose of ethambutol per body weight, duration of ethambutol therapy, cumulative dose of ethambutol, and cumulative dose of ethambutol per body weight).

Results The mean duration of ethambutol therapy was 3.6 months (range: 2-9 months) and the mean daily dose per body weight was $13.2 \mathrm{mg} / \mathrm{kg} /$ day. Analysis of response amplitude measures showed no significant difference in the mfERG N1 and P1 response amplitudes between the ethambutol and control groups at all ring eccentricities $(P>0.05)$. For implicit times, there were significant delays in the mfERG P1 implicit times of rings 4-6 in the ethambutol group compared with controls $(P=0.012$ to $P=0.041)$. Correlation analyses showed no significant correlation between the mfERG and ethambutol parameters $(P>0.05)$.

Conclusions The mfERG findings suggested that visually asymptomatic patients receiving ethambutol therapy might have localized mild electrophysiological changes involving the peripheral macula. These changes might be related to localized alteration of metabolism or

TYY Lai, JWS Ngai, RYK Lai and DSC Lam

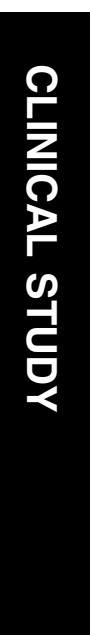

physiological changes associated with

ethambutol therapy.

Eye (2009) 23, 1707-1713; doi:10.1038/eye.2008.361;

published online 5 December 2008

Keywords: multifocal electroretinography; ethambutol; retinal dysfunction; drug toxicity

\section{Introduction}

Ethambutol is an effective drug in the first-line treatment of tuberculosis but its use is known to be associated with ocular toxicity. ${ }^{1}$ Toxic optic neuropathy is the most important ocular side effect and the incidence is related to the dosage and duration of ethambutol therapy. ${ }^{2,3}$ The incidence of ocular toxicity has been reported to be as high as $6 \%$ in patients receiving ethambutol of more than $15-25 \mathrm{mg} / \mathrm{kg} /$ day. $^{3,4}$ Ethambutol-associated optic neuropathy can be classified according to the visual field defect as central and peripheral types. The central type involves maculopapillary bundle and results in decreased visual acuity, centrocecal scotoma, and impairment in blue-yellow colour vision, whereas the peripheral type causes peripheral visual field constriction and red-green dyschromatopsia. ${ }^{5}$ In additiona to optic nerve toxicity, studies have also showed that ethambutol might also be toxic at the level of the retina. ${ }^{6-9}$

Multifocal electroretinography (mfERG) is an investigational tool, which allows the stimulation and recording of localized retinal responses from the macula topographically. ${ }^{10,11}$ Case reports have shown that mfERG can be used to detect the retinal functional abnormalities in patients with visual loss associated with ethambutol therapy. ${ }^{12-14}$ To further evaluate the mfERG changes in patients
Department of

Ophthalmology and Visual Sciences, The Chinese University of Hong Kong, Hong Kong Eye Hospital, Hong Kong, People's Republic of China

Correspondence: TYY Lai, Department of Ophthalmology and Visual Sciences,

The Chinese University of Hong Kong,

Hong Kong Eye Hospital, 147K Argyle Street, Kowloon, Hong Kong Tel: + (852) 27623153 ; Fax: + (852) 27159490.

E-mail: tyylai@

cuhk.edu.hk

Received: 7 August 2008 Accepted in revised form: 20 October 2008 Published online: 5 December 2008

Presented as an oral presentation at the 2008 annual meeting of the Association for Research in Vision and Ophthalmology (ARVO), Fort Lauderdale, FL, USA April-May 2008. Proprietary/financial interest: None 
taking ethambutol, we performed a study using mfERG to assess the retinal functional changes in visually asymptomatic patients receiving ethambutol therapy. We postulate that ethambutol might result in localized impairment of retinal function even in these visually asymptomatic patients.

\section{Materials and methods}

\section{Subjects}

This was a cross-sectional observational study carried out at the Department of Ophthalmology and Visual Sciences, the Chinese University of Hong Kong. Seventeen patients younger than 60 years of age receiving antituberculosis therapy with oral ethambutol were prospectively recruited. All patients were visually asymptomatic with visual acuity of $20 / 30$ or better and were able to cooperate for mfERG recordings. They also had normal colour vision, normal visual field on Humphrey 24-2 automated visual field testing, and normal fundus examination. Patients with myopia of more than $6 \mathrm{D}$ or any other concomitant eye diseases, such as glaucoma or diabetic retinopathy, were excluded. To prevent the potential influence of media opacity, such as cataract, in affecting the results, patients older than 60 years were excluded from the study. Informed consent was obtained in all patients and the study was approved by an institutional review board.

\section{MfERG testing technique}

MfERG recordings were performed using the VERIS 4.8 clinic system (Electro-Diagnostic Imaging, San Mateo, CA, USA) with reference to the ISCEV guidelines. ${ }^{15,16}$ Multiple retinal areas were stimulated using a stimulus array of 103 hexagons with a binary m-sequence length of $2^{14}$. The 103 hexagons were scaled by a stretch factor of 10.46, and the overall stimulus pattern subtended an angle of approximately $25^{\circ}$ on either side of fixation. The stimulus luminance was $200 \mathrm{~cd} / \mathrm{m}^{2}$ for the bright flashes and $1 \mathrm{~cd} / \mathrm{m}^{2}$ for the dark flashes. The stimulus was displayed using a 21-inch black and white monochrome cathode ray tube monitor at a frame rate of $75 \mathrm{~Hz}$. Before mfERG recording, patients' pupils were dilated using topical $0.5 \%$ tropicamide and $0.5 \%$ phenylephrine eye drops (Mydrin$\mathrm{P}$, Santen, Osaka, Japan), and all subjects were light adapted in room light for $15 \mathrm{~min}$ before testing. Recordings were divided into 16 segments and performed monocularly using Burian-Allen bipolar contact lens electrodes (Hansen Ophthalmic Laboratories, Iowa City, IA, USA). Topical anaesthesia with $0.5 \%$ proparacaine hydrochloride eye drops (Alcaine, Alcon, Forth Worth, TX, USA) was installed before the recording. Room lights were on throughout the recordings and the position of the patients' pupil was monitored using a refractor/camera system. Any segments with blinking, large eye movements, or fixation losses were discarded and recorded again. Retinal signals were filtered with low- and high-frequency cutoffs of 10 and $300 \mathrm{~Hz}$, respectively, and amplified with a gain of 100000 .

\section{Analysis of mfERG}

The first-order kernel of the mfERG responses was analysed. Individual mfERG responses for the 103 hexagons were grouped into six concentric rings centred on the fovea for analysis. The response amplitudes and implicit times of the first negative peak (N1) and the first positive peak (P1) of each individual ring were then measured. The N1 response amplitude was measured from the starting baseline to the base of the N1 trough and the P1 response amplitude from the N1 trough to the P1 peak. The N1 and P1 implicit times were measured from the stimulus onset to the N1 trough and P1 peak, respectively.

\section{Statistical analysis}

The mean first-order mfERG N1 and P1 response amplitudes and implicit times of the six concentric rings of the ethambutol group were compared with a normal control group of volunteers using the non-parametric Mann-Whitney U-test. Non-parametric Spearman's correlation analyses were also carried out to determine any potential correlations between the mfERG response amplitudes and implicit times of various rings and the ethambutol therapy parameters. These included the daily dose per body weight, the cumulative dose, the treatment duration, and the cumulative dose per body weight. Statistical analyses were carried out using SPSS 11.5, and a $P$-value of 0.05 was considered as statistically significant.

\section{Results}

\section{Patients' demographics}

Seventeen patients receiving ethambutol therapy were included in the study and only the data from the left eye were used for analysis. There were six male subjects and 11 female subjects. A total of 17 eyes of 17 age-similar control subjects were used as the control group. The mean \pm SD age of the ethambutol patients was $49.5 \pm 8.1$ years (range: $35-59$ years) and the mean \pm SD age of the control subjects was $46.9 \pm 6.5$ years (range:

38-58 years) (two-tailed $t$-test, $P=0.16$ compared with ethambutol group). The mean duration of ethambutol 
treatment was 3.6 months (range: 2-9 months) and the mean daily dose per body weight was $13.2 \mathrm{mg} / \mathrm{kg} /$ day (range: $6.9-25.5 \mathrm{mg} / \mathrm{kg} /$ day). Fourteen patients received antituberculosis therapy for pulmonary tuberculosis, two received treatment for lymph node tuberculosis infection, and one patient had treatment for vertebral tuberculosis. The demographic details of the patients are listed in Table 1.

\section{Changes in mfERG response amplitudes}

Figure 1 displays the mean N1 and P1 mfERG response amplitudes of the ethambutol patients compared with controls. Each round dot represents the mfERG response amplitude value of the individual eyes for N1 (Figure 1a) and $\mathrm{P} 1$ (Figure $1 \mathrm{~b}$ ). The boxes show the 25 and $75 \%$ range and the error bars represent the minimum to maximum values with the outliers excluded. Comparisons of the N1 and P1 mfERG response amplitudes showed no significant difference for all 6-ring eccentricities in the ethambutol group compared with controls $(P>0.05)$.

\section{Changes in mfERG implicit times}

The N1 and P1 implicit times of the ethambutol and control groups are displayed in Figure 2. Each round dot represents the mfERG implicit time value of the individual eyes for N1 (Figure 2a) and P1 (Figure 2b), with the boxes showing the 25 and $75 \%$ range. The error bars represent the minimum-to-maximum values excluding the outliers. Analyses showed no significant differences in the N1 implicit times of rings 1-6 and in the P1 implicit times of rings $1-3$ between the ethambutol and the control groups $(P>0.05)$. For the mean P1 implicit times of rings 4-6, significant delays were observed in patients receiving ethambutol compared with controls $(P=0.012-0.041)$.

\section{Correlation analyses}

Correlation analyses showed no significant correlations between the mfERG N1 and P1 response amplitudes and implicit times of all ring eccentricities with the ethambutol treatment parameters including daily dose per body weight of ethambutol, duration of ethambutol therapy, the cumulative dose of ethambutol therapy, and the cumulative dose of ethambutol per body weight $(P>0.05)$.

\section{Discussion}

Although visual loss associated with ethambutol is classically described as an optic neuropathy, various clinical and electrophysiological studies have shown that ethambutol might be toxic at various retinal cell layers. ${ }^{17}$ In a systematic review performed by Vistamehr et al, ${ }^{17}$ the authors summarized the clinical and electrophysiological findings of 101 cases of retinal toxicity associated with ethambutol in the literature. Reports have described patients receiving ethambutol therapy can develop retinal changes such as macular oedema, retinal pigment epithelial changes, retinal

Table 1 Demographic details of 17 patients receiving antituberculosis therapy with oral ethambutol

\begin{tabular}{|c|c|c|c|c|c|c|c|}
\hline Patient no. & Sex/age & $\begin{array}{l}\text { Site of tuberculosis } \\
\text { infection }\end{array}$ & $\begin{array}{c}\text { Daily dose of } \\
\text { ethambutol therapy } \\
\text { (mg/day) }\end{array}$ & $\begin{array}{c}\text { Daily adjusted } \\
\text { dose of ethambutol for } \\
\text { weight }(\mathrm{mg} / \mathrm{kg} / \text { day })\end{array}$ & $\begin{array}{c}\text { Duration of } \\
\text { ethambutol therapy } \\
\text { (months) }\end{array}$ & $\begin{array}{c}\text { Total cumulative } \\
\text { dose of } \\
\text { ethambutol }(g)\end{array}$ & $\begin{array}{l}\text { Other } \\
\text { antituberculosis } \\
\text { drugs }\end{array}$ \\
\hline 1 & $\mathrm{~F} / 50$ & Pulmonary & 1000 & 15.4 & 7 & 210 & INH, RIF, PZA \\
\hline 2 & $\mathrm{~F} / 53$ & Pulmonary & 1400 & 25.5 & 2 & 84 & INH, RIF, PZA \\
\hline 3 & $\mathrm{~F} / 55$ & Pulmonary & 700 & 12.8 & 2 & 42 & INH, RIF, PZA \\
\hline 4 & $\mathrm{M} / 48$ & Pulmonary & 500 & 7.1 & 2 & 30 & INH, RIF, PZA \\
\hline 5 & $\mathrm{~F} / 58$ & Lymph node & 800 & 13.3 & 2 & 48 & INH, RIF, PZA \\
\hline 6 & $\mathrm{~F} / 58$ & Lymph node & 700 & 9.3 & 3 & 63 & INH, RIF, PZA \\
\hline 7 & $\mathrm{~F} / 55$ & Pulmonary & 800 & 13.3 & 6 & 144 & RIF, PZA \\
\hline 8 & $\mathrm{M} / 57$ & Pulmonary & 800 & 14.6 & 2 & 48 & INH, RIF, PZA \\
\hline 9 & $\mathrm{M} / 51$ & Pulmonary & 450 & 6.9 & 3 & 41 & INH, RIF, PZA \\
\hline 10 & $\mathrm{~F} / 46$ & Pulmonary & 1000 & 16.7 & 9 & 270 & RIF, PZA \\
\hline 11 & $\mathrm{~F} / 59$ & Pulmonary & 1000 & 16.7 & 2 & 60 & INH, RIF, PZA \\
\hline 12 & $\mathrm{~F} / 58$ & Pulmonary & 700 & 9.3 & 6 & 126 & INH, RIF \\
\hline 13 & $\mathrm{M} / 40$ & Pulmonary & 1100 & 13.8 & 2 & 66 & INH, RIF, PZA \\
\hline 14 & $\mathrm{~F} / 42$ & Pulmonary & 800 & 16.0 & 2 & 48 & INH, RIF, PZA \\
\hline 15 & $\mathrm{M} / 38$ & Vertebral & 800 & 11.4 & 7 & 168 & INH, RIF, PZA \\
\hline 16 & $\mathrm{M} / 39$ & Pulmonary & 600 & 8.6 & 2 & 36 & INH, RIF, PZA \\
\hline 17 & $\mathrm{~F} / 35$ & Pulmonary & 800 & 14.6 & 2 & 48 & INH, RIF, PZA \\
\hline
\end{tabular}

$\mathrm{INH}=$ Isoniazid $; \mathrm{RIF}=$ Rifampicin $; \mathrm{PZA}=$ Pyrazinamide. 

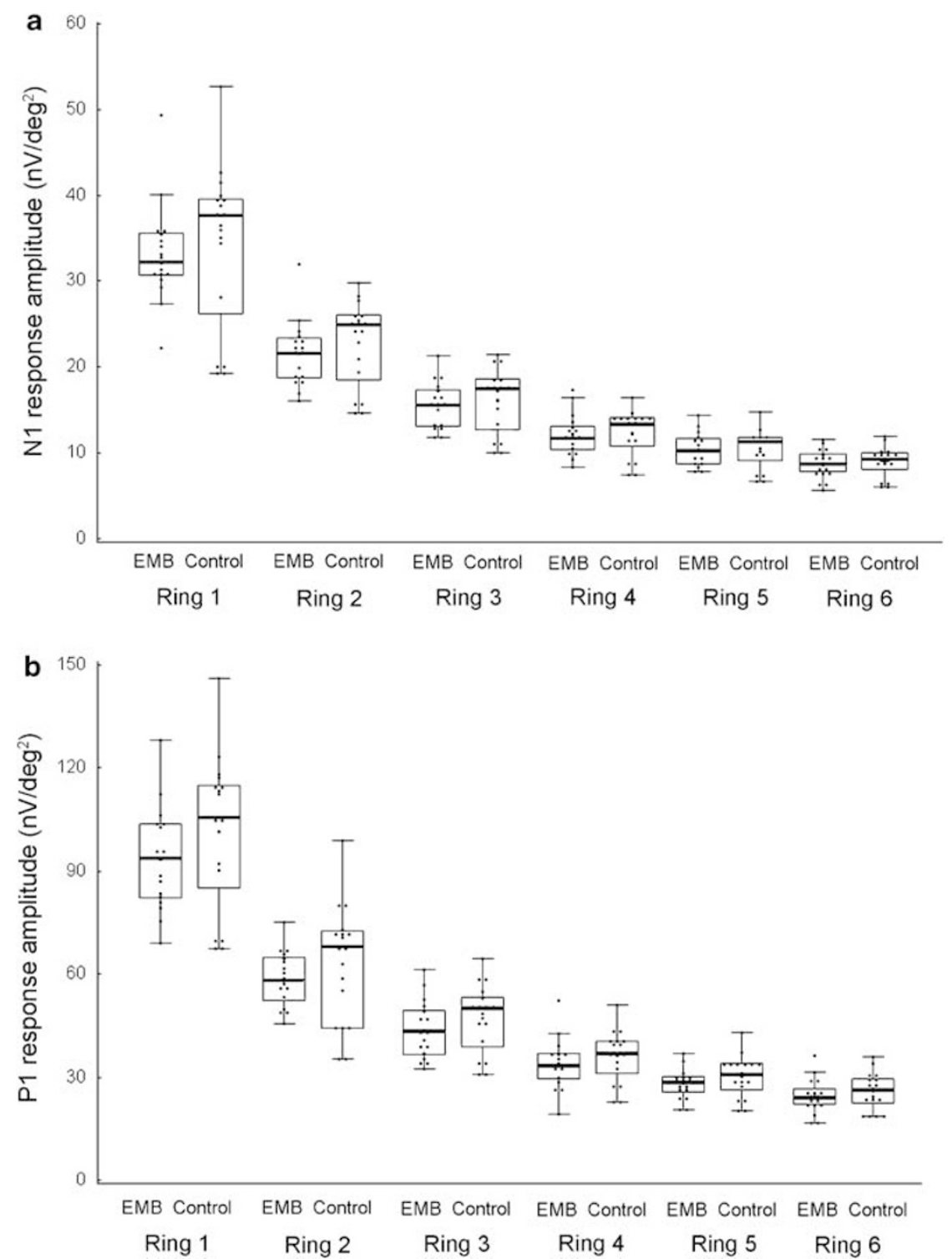

Figure 1 Box and whisker plots with individual values of (a) the N1 and (b) P1 mfERG response amplitude in the ethambutol and control groups. The boxes display the values from the lower to upper quartile and the bold horizontal lines represent median values. The error bars represent the minimum-to-maximum value excluding the outliers. No significant difference was found between the ethambutol and control groups for all ring eccentricities $(P>0.05)$.

haemorrhages, and retinal exudate. In addition, various electrophysiological abnormalities have been observed in patients using ethambutol. These changes include full-field ERG, pattern ERG, and electrooculography abnormalities. ${ }^{7,18,19}$ Laboratory experiments in rat retinal cell cultures have also shown that cellular damage with cytoplasmic vacuolar changes in neurons and astrocytes occurred following a 24-48-h exposure to ethambutol. ${ }^{20}$ These reactions appeared to be mediated by zinc and were reversible after exposure to ethambutol was removed. Studies using optical coherence tomography have also shown that ethambutol therapy is associated with reduction in the retinal nerve fibre layer thickness, suggestive of retinal ganglion cell loss. ${ }^{21,22}$ On the basis of these evidence, it is likely that ethambutol not only affects the optic nerve but also causes retinal changes, causing a neuroretinopathy.

As the ethambutol-associated retinopathy might be limited to the macula, case reports have used the mfERG to assess the macular dysfunction in patients with visual loss associated with ethambutol therapy. ${ }^{12-14}$ Behbehani et $a l^{13}$ described the mfERG findings of four patients with visual loss associated with ethambutol and it was found that patients had significantly lower N1 amplitude 

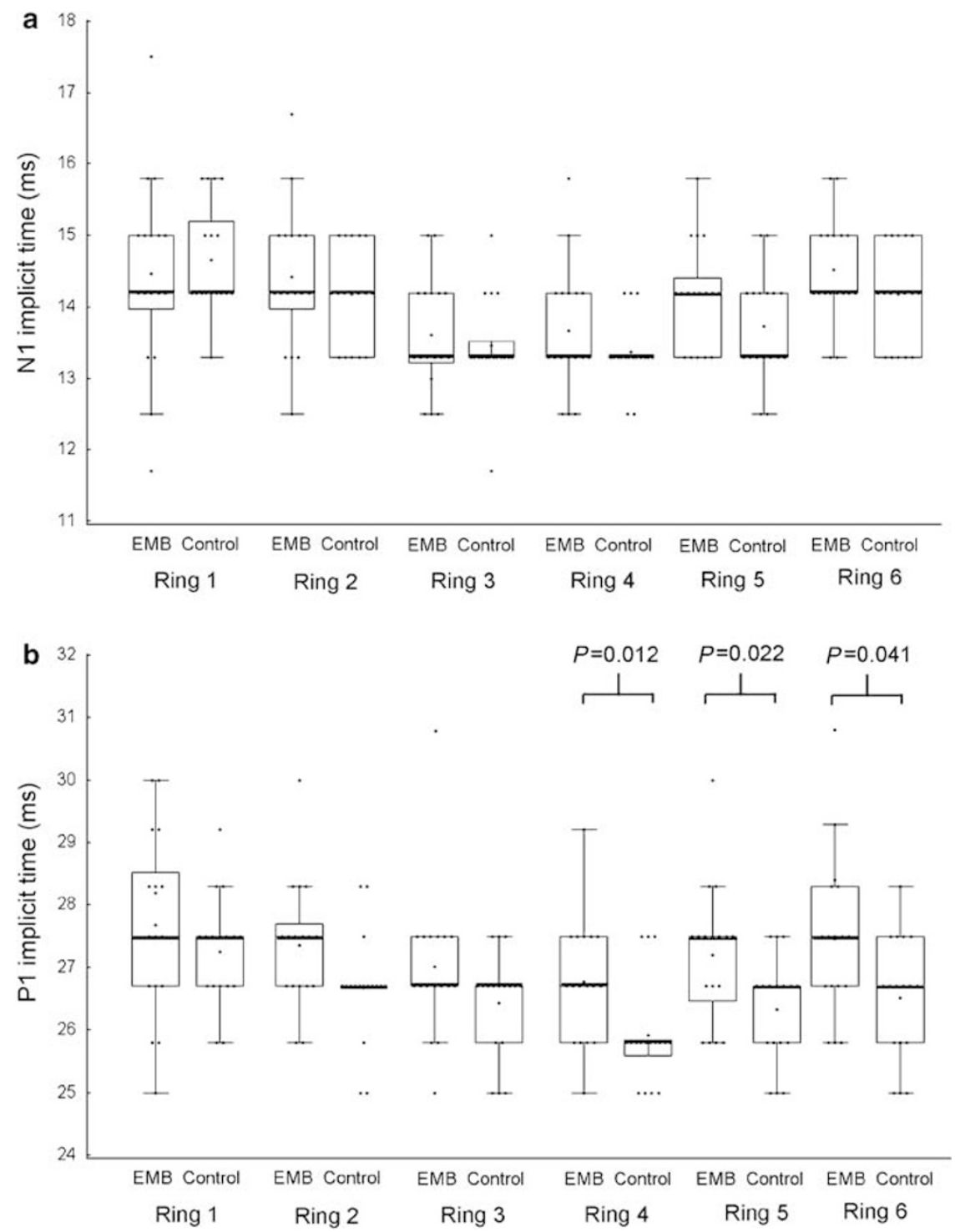

Figure 2 Box and whisker plots with individual values of (a) the N1 and (b) P1 mfERG implicit times in the ethambutol and control groups. The boxes display the values from the lower to upper quartile and the bold horizontal lines represent median values. The error bars represent the minimum-to-maximum value excluding the outliers. For the N1 mfERG implicit time, no significant difference was found between the ethambutol and control groups for all ring eccentricities $(P>0.05)$. For the P1 mfERG implicit time, significant delays were found in ethambutol patients compared with controls in rings $4-6(P<0.05)$.

compared with controls. Kardon et $a l^{14}$ and our earlier case report have also shown that the mfERG changes might be reversible after stopping of ethambutol therapy. ${ }^{12}$

In this study, we evaluated the mfERG changes in a group of visually asymptomatic patients taking ethambutol and compared the findings with a normal control group. In terms of the mfERG response amplitudes, no significant difference was found between the ethambutol and control groups. However, analysis of implicit times showed that small but statistically significant delays in P1 implicit times were observed in rings 4-6. This suggests that ethambutol use is associated with impairment in the peripheral macular function, whereas the central macular function was not affected. Earlier studies on mfERG have shown that the P1 response mainly arises from the on-bipolar and Müller cells, with some contributions from the retinal ganglion cells. ${ }^{23,24}$ These localized delays in mfERG P1 implicit times have also been observed in patients with diabetes mellitus or those using hydroxychloroquine in whom there were no visible retinal changes on clinical 
examination. ${ }^{24-26}$ We postulate that the delays in P1 implicit times observed in patients using ethambutol probably reflect physiological stress or localized alteration of cellular metabolism associated with ethambutol therapy. The exact cause for the delays in mfERG P1 implicit times in the ethambutol patients remained uncertain. Correlation analyses failed to show any correlation between the mfERG parameters with the daily dose per body weight, the duration of ethambutol therapy, the total cumulative dose of ethambutol, or the total cumulative dose of ethambutol per body weight on the mfERG parameters. The lack of correlation between the mfERG findings and the ethambutol parameters might be due to the small sample size in the study and further studies are required to determine the underlying mechanisms in causing the mfERG changes.

The main limitation of this study was its cross-sectional nature, and therefore we could not determine any possible serial changes in mfERG parameters associated with ethambutol therapy. Secondly, the magnitude of the implicit time changes observed was small in the region of around $1 \mathrm{~ms}$, and this small delay in implicit time might not be of clinical significance. Thirdly, the mfERG responses were grouped for analysis and localized impairment might be missed without analysing the responses from individual hexagons. Moreover, the small sample size in this study might have led to negative results in the analysis for the response amplitudes. Another reason for the lack of significant difference in mfERG response amplitudes might be due to the relatively short duration of ethambutol therapy in many patients. Fourthly, patients were also receiving other antituberculosis drugs including isoniazid and the additive toxic effects may also result in ocular toxicity, and therefore the contribution of other drugs in causing the mfERG changes observed could not be excluded. Lastly, there was a lack of anatomical correlation with the mfERG findings, such as optical coherence tomography, to document the retinal nerve fibre thickness, and only Humphrey 24-2 visual field testing was performed, which might have missed some central or cecocentral scotomas in the patients.

In conclusion, our findings suggested that slight delays in mfERG P1 implicit times of the peripheral macula may occur in patients on ethambutol therapy. Nonetheless, the other mfERG parameters were normal and there was no correlation between the mfERG P1 implicit time and the dosage or length of ethambutol use. Therefore, even though there was evidence suggesting that the drug is capable of producing retinal damage, it is difficult to base on our data to confirm that ethambutol resulted in significant macular dysfunction in visually asymptomatic patients at the doses specified. Further studies with larger sample sizes will be required to verify whether the mfERG changes observed were ethambutolrelated and to compensate the potential variability of mfERG amplitudes and implicit times. Longitudinal studies will also be beneficial to assess the clinical significance of the mfERG change in patients taking ethambutol and to determine whether mfERG is useful in predicting the development of ethambutol-associated neuroretinopathy.

\section{References}

1 Carr RE, Henkind P. Ocular manifestations of ethambutol. Toxic amblyopia after administration of an experimental antituberculous drug. Arch Ophthalmol 1962; 67: 566-571.

2 Leibold JE. The ocular toxicity of ethambutol and its relation to dose. Ann NY Acad Sci 1966; 135: 904-909.

3 Kumar A, Sandramouli S, Verma L, Tewari HK, Khosla PK. Ocular ethambutol toxicity: is it reversible? J Clin Neuroophthalmol 1993; 13: 15-17.

4 Alvarez KL, Krop LC. Ethambutol-induced ocular toxicity revisited. Ann Pharmacother 1993; 27: 102-103.

5 Schild HS, Fox BC. Rapid-onset reversible ocular toxicity from ethambutol therapy. Am J Med 1991; 90: 404-406.

6 Van Dijk BW, Spekreijse H. Ethambutol changes the color coding of carp retinal ganglion cells reversibly. Invest Ophthalmol Vis Sci 1983; 24: 128-133.

7 Kakisu Y, Adachi-Usami E, Mizota A. Pattern electroretinogram and visual evoked cortical potential in ethambutol optic neuropathy. Doc Ophthalmol 1987; 67: 327-334.

8 Nasemann J, Zrenner E, Riedel KG. Recovery after severe ethambutol intoxication: psychophysical and electrophysiological correlations. Doc Ophthalmol 1989; 71: 279-292.

9 Heng JE, Vorwerk CK, Lessell E, Zurakowski D, Levin LA, Dreyer EB. Ethambutol is toxic to retinal ganglion cells via an excitotoxic pathway. Invest Ophthalmol Vis Sci 1999; 40: 190-196.

10 Sutter EE, Tran D. The field topography of ERG components in man: I. The photopic luminance response. Vision Res 1992; 32: 433-436.

11 Lai TY, Chan WM, Lai RY, Ngai JW, Li H, Lam DS. The clinical applications of multifocal electroretinography: a systematic review. Surv Ophthalmol 2007; 52: 61-96.

12 Lai TY, Chan WM, Lam DS, Lim E. Multifocal electroretinogram demonstrated macular toxicity associated with ethambutol related optic neuropathy. $\mathrm{Br} J$ Ophthalmol 2005; 89: 774-775.

13 Behbehani RS, Affel EL, Sergott RC, Savino PJ. Multifocal ERG in ethambutol associated visual loss. $\mathrm{Br} J$ Ophthalmol 2005; 89: 976-982.

14 Kardon RH, Morrisey MC, Lee AG. Abnormal multifocal electroretinogram (mfERG) in ethambutol toxicity. Semin Ophthalmol 2006; 21: 215-222.

15 Marmor MF, Hood DC, Keating D, Kondo M, Seeliger MW, Miyake Y. International society for clinical electrophysiology of vision. Guidelines for basic multifocal electroretinography (mfERG). Doc Ophthalmol 2003; 106: 105-115.

16 Hood DC, Bach M, Brigell M, Keating D, Kondo M, Lyons JS et al. ISCEV guidelines for clinical multifocal 
electroretinography (2007 edition). Doc Ophthalmol 2008; 116: 1-11.

17 Vistamehr S, Walsh TJ, Adelman RA. Ethambutol neuroretinopathy. Semin Ophthalmol 2007; 22: 141-146.

18 Hennekes R. Clinical ERG findings in ethambutol intoxication. Graefes Arch Clin Exp Ophthalmol 1992; 218 318-321.

19 Yen MY, Wang AG, Chiang SC, Liu JH. Ethambutol retinal toxicity: an electrophysiology study. J Formos Med Assoc 2000; 99: 630-634.

20 Yoon YH, Jung KH, Sadun AA, Shin HC, Koh JY. Ethambutol-induced vacuolar changes and neuronal loss in rat retinal cell culture: mediated by endogenous zinc. Toxicol Appl Pharmacol 2000; 162: 107-114.

21 Zoumalan CI, Agarwal M, Sadun AA. Optical coherence tomography can measure axonal loss in patients with ethambutol-induced optic neuropathy. Graefes Arch Clin Exp Ophthalmol 2005; 243: 410-416.
22 Chai SJ, Foroozan R. Decreased retinal nerve fibre thickness detected by optical coherence tomography in patients with ethambutol-induced optic neuropathy. Br J Ophthalmol 2007; 91: 895-897.

23 Hood DC, Seiple W, Holopigian K, Greenstein V. A comparison of the components of the multifocal and full-field ERGs. Vis Neurosci 1997; 14: 533-544.

24 Fortune B, Schneck ME, Adams AJ. Multifocal electroretinogram delays reveal local retinal dysfunction in early diabetic retinopathy. Invest Ophthalmol Vis Sci 1999; 40: 2638-2651.

25 Lai TY, Chan WM, Li H, Lai RY, Lam DS. Multifocal electroretinographic changes in patients receiving hydroxychloroquine therapy. Am J Ophthalmol 2005; 140: 794-807.

26 Kim SJ, Song SJ, Yu HG. Multifocal electroretinogram responses of the clinically normal retinal areas in diabetes. Ophthalmic Res 2007; 39: 282-288. 